\title{
Advanced Image Processing for Defense and Security Applications
}

\author{
Eliza Yingzi Du, ${ }^{1}$ Robert Ives, ${ }^{2}$ Alan van Nevel, ${ }^{3}$ and Jin-Hua She ${ }^{4}$ \\ ${ }^{1}$ Department of Electrical and Computer Engineering, Indiana University-Purdue University Indianapolis, \\ 723W. Michigan Street, SL 160, Indainapolis, IN 46259, USA \\ ${ }^{2}$ Department of Electrical Engineering, US Naval Academy, 105 Maryland Avenue, MS 14B, Annapolis, MD 21402, USA \\ ${ }^{3}$ Image and Signal Processing Branch, Research Department, Naval Air Warfare Center, 1900 N Knox Road, M/S 6302, \\ China Lake, CA 93555, USA \\ ${ }^{4}$ School of Computer Science, Tokyo University of Technology, 1404-1 Katakura, Hachioji, Tokyo 192-0982, Japan
}

Correspondence should be addressed to Eliza Yingzi Du, yidu@iupui.edu

Received 31 December 2010; Accepted 31 December 2010

Copyright ( 2010 Eliza Yingzi Du et al. This is an open access article distributed under the Creative Commons Attribution License, which permits unrestricted use, distribution, and reproduction in any medium, provided the original work is properly cited.

The history of digital image processing can be traced back to the 1920s when digital images were transferred between London and New York. However, in the past, the cost of processing was very high because the imaging sensors and computational equipments were very expensive and had only limited functions. As a result, the development of digital image processing was limited.

As optics, imaging sensors, and computational technology advanced, image processing has become more commonly used in many different areas. Some areas of application of digital image processing include image enhancement for better human perception, image compression and transmission, as well as image representation for automatic machine perception.

Most notably, digital image processing has been widely deployed for defense and security applications such as small target detection and tracking, missile guidance, vehicle navigation, wide area surveillance, and automatic/aided target recognition. One goal for an image processing approach in defense and security applications is to reduce the workload of human analysts in order to cope with the ever increasing volume of image data that is being collected. A second, more challenging goal for image processing researchers is to develop algorithms and approaches that will significantly aid the development of fully autonomous systems capable of decisions and actions based on all sensor inputs.

For this special issue, our aim is to bring together researchers designing or developing advanced image processing techniques/systems, with a particular emphasis on defense and security applications. 105 works, from 12 countries (Canada, Taiwan, Spain, China, Egypt, Iran, France, Italy, Slovenia, Japan, and USA) were submitted. Then, 44 works were accepted for publication after being thoroughly reviewed by international experts on subject matter areas. In this special issue, we have discussed following topics: information assurance (including watermarking and visual secret sharing scheme), steganography, target detection and tracking (including abnormal event detection, human detection and tracking, vehicle trajectory estimation, radar and 3D Radar image processing, multisensory surveillance, hyperspectral image processing, obstacle detection, ISAR image processing, sonar image processing, 3D shape retrieval and image analysis, and image segmentation), and biometrics (including iris recognition, face recognition, hardware design for biometrics, and multicliet identification).

Eliza Yingzi Du Robert Ives Alan van Nevel Jin-Hua She 